\title{
Two Lemmas in Łocal Analytic Geometry
}

\author{
Charles L. Epstein and Gennadi M. Henkin \\ Department of Mathematics, University of Pennsylvania \\ and \\ University of Paris, VI \\ This paper is dedicated to Leon Ehrenpreis.
}

\begin{abstract}
We prove two results about the local properties of generically one to one analytic
\end{abstract} mappings.

Version: .76; Revised: 12-15-98; Run: March 23, 1999

\section{§1: INTRODUCTION}

In this paper we consider two local properties of analytic maps which are generically one to one. First we consider holomorphic maps from open sets in $\mathbb{C}^{2}$ which behave locally like monoidal transformations: Let $(z, w)$ denote coordinates for $\mathbb{C}^{2}$ and $D, D^{\prime} \subset \mathbb{C}^{2}$ be neighborhoods of $(0,0)$. We call a holomorphic map $f: D \rightarrow D^{\prime}$ a germ of a blowdown if

(1) $f(0, w)=(0,0)$,

(2) $f$ is injective on $D \backslash\{z=0\}$.

We prove the following normal form result for such maps:

Lemma 1. Suppose that $f: D \rightarrow D^{\prime}$ is a germ of a blowdown then there are local coordinates, $(\zeta, \xi)$ on a neighborhood of $(0,0)$ such that in these coordinates the map is either

$$
\begin{gathered}
f(\zeta, \xi)=\left(\zeta, \zeta^{k} \xi\right), \quad k \in \mathbb{N} \text { or } \\
f(\zeta, \xi)=\left(\zeta^{j}, \zeta^{k_{1}}\left(\alpha_{1}+\zeta^{k_{2}}\left(\alpha_{2}+\ldots \zeta^{k_{p}}\left(\alpha_{p}+\xi\right) \ldots\right)\right),\right. \\
\alpha_{i} \in \mathbb{C}, k_{i} \in \mathbb{N}, i=1, \ldots, p .
\end{gathered}
$$

As a consequence of the lemma we obtain the following:

Key words and phrases. blow down, Castelnuovo, Łojasiewicz, injectvity bound.

Research supported in part by the University of Paris, VI and NSF grant DMS96-23040

Typeset by $\mathcal{A} \mathcal{M} \mathcal{S}-\mathrm{T}_{\mathrm{E}} \mathrm{X}$ 
Corollary 1. If $f: D \rightarrow D^{\prime}$ is a germ of a blowdown then there is a finite sequence of point blow-ups of

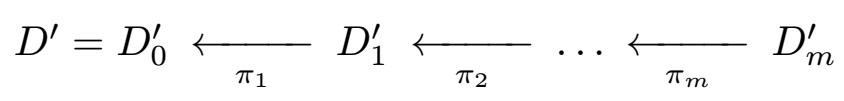

and lifted maps $f_{i}: D \rightarrow D_{i}^{\prime}$ so that

$$
f=\pi_{1} \circ \cdots \circ \pi_{i} \circ f_{i}
$$

and $f_{m}: D \rightarrow D_{m}^{\prime}$ is a germ of a biholomorphism.

This result shows that Castelnuovo's classical result characterizing a generically one to one map between smooth, compact complex surfaces as a composition of monoidal transformations has a completely satisfactory local analogue, see [GrHa].

The other result we prove is a consequence of the Łojasiewicz inequality. We let $|x|$ denote the Euclidean norm in $\mathbb{R}^{m}$ for any $m$. If $F \subset \mathbb{R}^{m}$ we let

$$
d(x, F)=\inf _{y \in F}|x-y| .
$$

Let $f$ be a real analytic function in an open neighborhood, $\Omega \subset \mathbb{R}^{n}$ of 0 and suppose that $f(0)=0$. Let $Z_{f}$ denote the zero locus of $f$. Lojasiewicz proved that for each compact subset $K \subset \Omega$ there exist positive constants, $C, N$ so that

$$
|f(x)| \geq C\left[d\left(x, Z_{f}\right)\right]^{N},
$$

see [Ło]. Hörmander proved a similar result but assuming that $f$ is a polynomial. These results were originally used to prove division theorems for distributions. Hömander used his result to prove a tempered version of the Malgrange-Ehrenpreis theorem, see [Hö] and [Eh,Ma].

Now suppose that $D_{r} \subset \mathbb{R}^{n}$ is the ball of radius $r$ and that $\psi: D_{1+\epsilon} \rightarrow \mathbb{R}^{N}, \epsilon>0$ is a real analytic mapping. Let $E_{\psi}=\left\{x \in D_{1+\epsilon} \mid \operatorname{rank} \psi_{*}<n\right\}$.

Lemma 2. Suppose that $\psi$ is as above and that $E_{\psi}$ is a non-empty proper subvariety of $D_{1+\epsilon}$. Suppose further that $\psi$ is one to one on $D_{1+\epsilon} \backslash E_{\psi}$ and $\psi\left(D_{1+\epsilon} \backslash E_{\psi}\right) \cap \psi\left(E_{\psi}\right)=\emptyset$. Then there exist positive constants, $C, N$ so that

$$
|\psi(x)-\psi(y)| \geq C|x-y|\left[d\left(x, E_{\psi}\right)+d\left(y, E_{\psi}\right)\right]^{N} \text { for } x, y \in D_{1}
$$

We have found this a useful consequence of the Lojasiewicz inequality. For example we have the following corollary:

Corollary 2. With $\psi$ as in Theorem 2 there exists an $M>0$ such that if $f \in \operatorname{Lip}_{1}\left(D_{1}\right)$ and

$$
|\nabla f(x)| \leq C\left[d\left(x, E_{\psi}\right)\right]^{M}
$$

then $\psi_{*}(f)(y)=f\left(\psi^{-1}(y)\right)$ is a Lipschitz function on the closed set $\psi\left(D_{1}\right)$. There is a constant $C^{\prime}$, independent of $f$ such that

$$
\left\|\psi_{*}(f)\right\|_{\operatorname{Lip}_{1}\left(\psi\left(D_{1}\right)\right)} \leq C^{\prime}\left(\|f\|_{C_{0}\left(D_{1}\right)}+\sup _{x \in D_{1}} \frac{|\nabla f(x)|}{\left[d\left(x, E_{\psi}\right)\right]^{M}}\right) .
$$

The proof of this corollary can be found in [EpHe]. 


\section{$\S 2:$ Proof of Lemma 1}

Proof of Lemma 1. Since $f$ maps $\{z=0\}$ to $(0,0)$ there are positive integers, $j, k$ such that

$$
f(z, w)=\left(z^{j} \varphi_{1}(z, w), z^{k} \varphi_{2}(z, w)\right)
$$

where $\varphi_{1}, \varphi_{2}$ are holomorphic. We suppose that $j, k$ are the maximal such integers. Define the varieties:

$$
V_{i}=\left\{\varphi_{i}^{-1}(0)\right\}, i=1,2 .
$$

By assumption $V_{i} \cap\{z=0\}$ is a finite set for $i=1,2$. We claim that $(0,0) \notin V_{i}$ for at least one value of $i$.

Suppose that this is not the case. Each irreducible component of $V_{2}$ has a local parametrization of the form $t \rightarrow\left(t^{a} g(t), t^{b} h(t)\right)$. This map is injective in some neighborhood of $t=0$ and $a$ and $b$ are positive integers. In particular there is an $\epsilon>0$ such that $g(t) \neq 0$ for $|t|<\epsilon$. We consider the composition

$$
f\left(t^{a} g(t), t^{b} h(t)\right)=\left(t^{j a} g(t)^{j} \varphi_{1}\left(t^{a} g(t), t^{b}(h(t)), 0\right) .\right.
$$

Since $\varphi_{1}(0,0)=0$ there is a $c>0$ such that

$$
\varphi_{1}\left(t^{a} g(t), t^{b}(h(t))=t^{c} k(t), \text { where } k(0) \neq 0 .\right.
$$

By assumption this map is injective in a neighborhood of 0 , on the other hand it has the form:

$$
f\left(t^{a} g(t), t^{b} h(t)\right)=\left(t^{j a+c} g(t)^{j} k(t), 0\right)
$$

From this we conclude that $j a+c=1$. However this contradicts the assumptions that $j, a$ and $c$ are all at least 1 . From this the claim follows. We therefore assume that $\varphi_{1}(0,0) \neq 0$. If we introduce the new coordinate

$$
\zeta=z\left(\varphi_{1}(z, w)\right)^{\frac{1}{j}}
$$

then in a neighborhood of $(0,0)$ the map has the form:

$$
f(\zeta, w)=\left(\zeta^{j}, \zeta^{k} \varphi_{2}(\zeta, w)\right) .
$$

We first dispose of a simple special case. If $\varphi_{2}(0,0)=0$ then using the local parametrization of $V_{2}$ from above we conclude that $j a=1$. In particular $j=1$. Suppose that $\varphi_{2}(\zeta, 0)=0$ so that $\varphi_{2}=w^{l} \varphi_{2}^{\prime}(\zeta, w)$ for an $l>0$; where this is the maximal such $l$. For sufficiently small $\zeta \neq 0$ the map

$$
w \rightarrow w^{l} \varphi_{2}^{\prime}(\zeta, w)
$$

must be injective and therefore $l=1$. Finally we observe that if $\varphi_{2}^{\prime}(0,0)=0$ then the set $f^{-1}(\{(t, 0)\}) \backslash\{(0,0)\}$ would have two distinct components passing through $(0,0)$. As this would contradict the injectivity of $f$ on the complement of $\{z=0\}$ it follows that $\varphi_{2}^{\prime}(0,0) \neq 0$. If we introduce, as second coordinate $\xi=w \varphi_{2}^{\prime}(\zeta, w)$, then the map takes the form

$$
f(\zeta, \xi)=\left(\zeta, \zeta^{k} \xi\right)
$$


Now we treat the general case. There is a fixed $\epsilon>0$ such that the maps $\left\{w \rightarrow \varphi_{2}(\zeta, w)\right.$ : $0<|\zeta|<\epsilon\}$ are injective in the set $B_{\epsilon}=\{|w|<\epsilon\}$. Hurwitz's theorem implies that $w \rightarrow$ $\varphi_{2}(0, w)$ is either injective in $B_{\epsilon}$ or constant. Let

$$
\alpha_{1}=\varphi_{2}(0,0)
$$

In the former case we introduce the new coordinate $\xi=\varphi_{2}(\zeta, w)-\alpha_{1}$ which puts the map into the normal form (1.2):

$$
f(\zeta, \xi)=\left(\zeta^{j}, \zeta^{k}\left(\alpha_{1}+\xi\right)\right)
$$

Note that if $\alpha_{1}=0$ then $j=1$ as follows from the argument above.

In the latter case we set $k_{1}=k$ and let $0<k_{2}$ be the largest integer such that

$$
\varphi_{2}(\zeta, w)=\alpha_{1}+\zeta^{k_{2}} \varphi_{2}^{(1)}(\zeta, w)
$$

where $\varphi_{2}^{(1)}$ is a holomorphic function. The same observation applies in this case: the maps $\left\{w \rightarrow \varphi_{2}^{(1)}(\zeta, w): 0<|\zeta|<\epsilon\right\}$ are injective in the set $B_{\epsilon}$. This leads to the same dichotomy: either $\varphi_{2}^{(1)}(0, w)$ is injective on $B_{\epsilon}$ or constant. If we repeat this argument $p$-times we obtain sequences of complex numbers, $\left\{\alpha_{1}, \ldots, \alpha_{p}\right\}$, positive integers, $\left\{k_{1}, \ldots, k_{p+1}\right\}$ and a holomorphic function, $\varphi_{2}^{(p)}$ so that

$$
\varphi_{2}(\zeta, w)=\zeta^{k_{1}}\left(\alpha_{1}+\zeta^{k_{2}}\left(\alpha_{2}+\zeta^{k_{3}}\left(\ldots\left(\alpha_{p}+\zeta^{k_{p+1}} \varphi_{2}^{(p)}\right) \ldots\right)\right.\right.
$$

As before the maps $w \rightarrow \varphi_{2}^{(p)}(\zeta, w)$ are injective in $B_{\epsilon}$ for $\zeta \neq 0$. Observe that $\partial_{w} \varphi_{2}(\zeta, w)$ is divisible by $\zeta^{p+1}$. In order for $\varphi_{2}$ to depend on $w$ in a non-trivial way there must a finite value, $p$ such that $w \rightarrow \varphi_{2}^{(p)}(0, w)$ is injective in $B_{\epsilon}$. If, for this $p$ we let $\xi=\varphi_{2}^{(p)}(\zeta, w)-\alpha_{p+1}$ then we obtain the normal form, (1.2) for $f$ :

$$
f(\zeta, \xi)=\left(\zeta^{j}, \zeta^{k_{1}}\left(\alpha_{1}+\zeta^{k_{2}}\left(\cdots+\zeta^{k_{p+1}}\left(\alpha_{p+1}+\xi\right) \ldots\right)\right)\right.
$$

Remark. The normal form, (1.2) can be re-expressed as

$$
f(\zeta, \xi)=\left(\zeta^{j}, q(\zeta)+\zeta^{N} \xi\right)
$$

where $q(\zeta)$ is a polynomial of degree at most $N=k_{1}+\cdots+k_{p}$. The condition that $f$ be injective in some deleted neighborhood of $(0,0)$ is: for each $j^{\text {th }}$ root of unity, $e^{i \omega}$ the polynomial $q(\zeta)-q\left(e^{i \omega} \zeta\right)$ is not divisible by $\zeta^{N}$. For example, if $\alpha_{p}=0$ so that the degree of $q$ is less than $N$ this is equivalent to the condition:

$$
\operatorname{gcd}\left(j, k_{1}, k_{1}+k_{2}, \ldots, k_{1}+\ldots k_{p}\right)=1
$$

We now deduce the corollary: 
Proof of Corollary 1. The proof is a simple recursive argument using the normal form and the fact that a blow-up is locally described by either $(z, w) \rightarrow\left(z, \frac{w}{z}\right)$ or $(z, w) \rightarrow\left(\frac{z}{w}, w\right)$. If the map takes the normal form (1.1) then blowing up the origin in the target $k$-times and lifting the map $f$ each time leads to a space $D_{k}^{\prime}$ and a map $f_{k}: D \rightarrow D_{k}^{\prime}$, given by: $f_{k}(\zeta, \xi)=(\zeta, \xi)$.

If the map takes the normal form, (1.2) then the Jacobian determinant, $J_{f}$ is easily computed, it is

$$
J_{f}=j \zeta^{j+k_{1}+\cdots+k_{p}-1} .
$$

Let ord $J_{f}$ denote the order of vanishing of $J_{f}$ along $\{z=0\} \cap D$, this is of course invariant under biholomorphisms. We obtain a sequence of spaces, $\left\{D_{j}^{\prime}\right\}$ by the prescription: Define $\pi_{1}: D_{1}^{\prime} \rightarrow D^{\prime}$ as the blow-up of $f(\{z=0\} \cap D)=(0,0)$. The map, $f$ lifts to define a map,

$$
f_{1}: D \longrightarrow D_{1}^{\prime}
$$

which satisfies $f=\pi_{1} \circ f_{1}$. It is evident that ord $J_{f_{1}}<$ ord $J_{f}$. If $J_{f_{1}}$ is non-vanishing then we are done as $f_{1}$ is then the germ of a biholomorphism. Otherwise $f_{1}: D \rightarrow D_{1}^{\prime}$ is the germ of a blow-down but $f_{1}(\{\zeta=0\} \cap D)$ may not be $(0,0)$. The normal form theorem applies mutatis mutandis to this case as well and so we can define $D_{2}^{\prime}$ by blowing up $f_{1}(\{\zeta=0\} \cap D)$ to obtain $\pi_{2}: D_{2}^{\prime} \rightarrow D_{1}^{\prime}$ and $f_{2}: D \rightarrow D_{2}^{\prime}$ with $f_{1}=\pi_{2} \circ f_{2}$. Apply this process recursively: assume that we have obtained spaces,

$$
D_{k}^{\prime} \stackrel{\pi_{k}}{\longrightarrow} D_{k-1}^{\prime} \stackrel{\pi_{k-1}}{\longrightarrow} \ldots \stackrel{\pi_{1}}{\longrightarrow} D^{\prime}
$$

and germs of blow-downs

$$
f_{i}: D \rightarrow D_{i}^{\prime} \text { with } f_{i}=\pi_{i} \circ f_{i-1}, i=1 \ldots, k .
$$

The space $D_{i}^{\prime}$ is obtained by blowing up $f_{i-1}(\{\zeta=0\} \cap D)$. At each step we see that ord $J_{f_{i}}<$ ord $J_{f_{i-1}}$. If ord $J_{f_{k}}>0$ then $f_{k}$ is the germ of a blow-down and we define $\left(D_{k+1}^{\prime}, f_{k+1}\right)$ as above otherwise $f_{k}$ is the germ of a biholomorphism. With each blow-up the ord $J_{f_{k}}$ decreases by at least 1 thus this process must terminate after finitely steps.

\section{§3: Proof of Lemma 2}

Proof of Lemma 2. In this argument $\psi: D_{1} \rightarrow \mathbb{R}^{N}$ is a real analytic map defined and satisfying the hypotheses of the theorem on a neighborhood of $\bar{D}_{1}$. We let $z$ and $w$ denote points in $\bar{D}_{1}$. First consider the real analytic function:

$$
\rho(z, w)=|\psi(z)-\psi(w)|^{2} .
$$

This function vanishes on $Z_{\rho} \subset \Delta \cup E_{\psi} \times E_{\psi}$ where

$$
\Delta=\left\{(p, p) \mid p \in D_{1}\right\} \text { and } E_{\psi}=\left\{z \in D_{1} \mid \operatorname{rank} \psi_{*}<n\right\} .
$$

We get containment and not equality whenever $E_{\psi}$ has several connected components. Apply Łojasiewicz' inequality to obtain positive constants $C_{1}, N_{1}$ so that

$$
\rho(z, w) \geq \underset{5}{C_{1}\left[d\left((z, w), Z_{\rho}\right)\right]^{N_{1}} .}
$$


Evidently

$$
d\left((z, w), Z_{\rho}\right) \geq \min \left\{\frac{1}{2}|z-w|,\left[d\left(z, E_{\psi}\right)^{2}+d\left(w, E_{\psi}\right)^{2}\right]^{\frac{1}{2}}\right\} .
$$

If $d((z, w), \Delta) \geq d\left((z, w), E_{\psi} \times E_{\psi}\right)$ then (3.2) implies that

$$
\rho(z, w) \geq C_{1}^{\prime}|z-w|\left[d\left(z, E_{\psi}\right)+d\left(w, E_{\psi}\right)\right]^{N_{1}},
$$

for some possibly smaller constant. For $\delta>0, L \geq 1$ we define the sets:

$$
A_{\delta, L}=\left\{(z, w) \mid \quad d((z, w), \Delta) \geq \delta\left[d\left(z, E_{\psi}\right)+d\left(w, E_{\psi}\right)\right]^{L}\right\} .
$$

It follows from (3.2) that we have the estimates

$$
\rho(z, w) \geq C_{1}^{\prime}|z-w|\left[d\left(z, E_{\psi}\right)+d\left(w, E_{\psi}\right)\right]^{L\left(N_{1}-1\right)} \text { for }(z, w) \in A_{\delta, L} .
$$

We are left to consider the set

$$
B_{\delta, L}=\left\{(z, w)|\quad| z-w \mid<\delta\left[d\left(z, E_{\psi}\right)+d\left(w, E_{\psi}\right)\right]^{L}\right\}
$$

for a $\delta>0$ and $L \geq 1$ which are yet to be determined. To that end we express

$$
\psi(z)-\psi(w)=M(z, w)(z-w)
$$

where $M(z, w)$ is the $N \times n$ matrix valued real analytic function given by:

$$
M(z, w)=\int_{0}^{1} \nabla \psi(t z+(1-t) w) d t .
$$

Observe that

$$
M(z, z)=\nabla \psi(z)
$$

Let $\mathcal{I}_{n}$ denote set of multi-indices

$$
\mathcal{I}_{n}=\left\{\left(i_{1}, \ldots, i_{n}\right) \mid \quad 1 \leq i_{1}<\ldots, i_{n} \leq N\right\} .
$$

If we let $\underline{i}=\left(i_{1}, \ldots, i_{n}\right)$ denote an element of $\mathcal{I}_{n}$ then $M_{\underline{i}}$ is the $n \times n$ sub-matrix

$$
M_{\underline{i}}=\left(\begin{array}{ccc}
M_{i_{1} 1} & \ldots & M_{i_{1} n} \\
\vdots & & \vdots \\
M_{i_{n} 1} & \ldots & M_{i_{n} n} \\
6 & &
\end{array}\right)
$$


and $\psi_{\underline{i}}$ the $n$-vector valued function

$$
\psi_{\underline{i}}(z)=\left(\begin{array}{c}
\psi_{i_{1}}(z) \\
\vdots \\
\psi_{i_{n}}(z)
\end{array}\right) .
$$

For each such multi-index we have the identity:

$$
\psi_{\underline{i}}(z)-\psi_{\underline{i}}(w)=M_{\underline{i}}(z, w)(z-w) .
$$

If $\operatorname{det} M_{\underline{i}}(z, w) \neq 0$ then it follows easily from the fact that $\psi$ is smooth, Cramer's rule and the Cauchy-Schwarz inequality that there is a positive constant, $C_{4}$ such that

$$
\left|\psi_{\underline{i}}(z)-\psi_{\underline{i}}(w)\right| \geq C_{4}\left|\operatorname{det} M_{\underline{i}}(z, w)\right||z-w| .
$$

Note that $C_{4}>0$ is a fixed constant which is independent of $z, w$ and $\underline{i}$.

Define the real analytic function

$$
m(z, w)=\sum_{\underline{i} \in \mathcal{I}_{n}}\left|\operatorname{det} M_{\underline{i}}(z, w)\right|^{2} .
$$

From (3.6) and the hypothesis of the theorem it follows that $m(z, z)$ vanishes exactly on the set $E_{\psi}$. Thus we can apply Łojasiewicz' inequality to obtain that there exist positive constants, $C_{5}, N_{2}$ such that

$$
m(z, z) \geq C_{5}\left[d\left(z, E_{\psi}\right)\right]^{N_{2}} .
$$

For an $L>N_{2}$ and $\delta>0$, sufficiently small we now show that there exists a constant $C_{5}^{\prime}>0$ such that

$$
m(z, w)>C_{5}^{\prime}\left[d\left(z, E_{\psi}\right)+d\left(w, E_{\psi}\right)\right]^{N_{2}} \text { for }(z, w) \in B_{\delta, L} .
$$

It follows from the smoothness of $m(z, w)$ and the mean value inequality that there exists a constant, $C_{6}$ so that

$$
m(z, w) \geq m(z, z)-C_{6}|z-w|
$$

hence (3.9) implies that

$$
m(z, w) \geq C_{5}\left[d\left(z, E_{\psi}\right)\right]^{N_{2}}-C_{6} \delta\left[d\left(z, E_{\psi}\right)+d\left(w, E_{\psi}\right)\right]^{L} \text { for }(z, w) \in B_{\delta, L} .
$$

If $(z, w) \in B_{\delta, L}$ then the triangle inequality implies that

$$
d\left(w, E_{\psi}\right)-d\left(z, E_{\psi}\right) \leq d(w, z) \leq \delta\left[d\left(z, E_{\psi}\right)+d\left(w, E_{\psi}\right)\right]^{L} .
$$

For sufficiently small $\delta>0$, this estimate and the binomial theorem imply that there exists a positive constant $C_{7}$ so that

$$
\frac{1}{C_{7}} d\left(z, E_{\psi}\right) \leq d\left(w, E_{\psi}\right) \leq C_{7} d\left(z, E_{\psi}\right) .
$$

Putting together (3.11) and (3.12) we obtain (3.10). Let $K$ be the cardinality of $\mathcal{I}_{n}$. Then (3.10) implies that for each $(z, w) \in B_{\delta, L}$ there exists a $\underline{i} \in \mathcal{I}_{n}$ for which we have the estimate:

$$
\left|\operatorname{det} M_{\underline{i}}(z, w)\right|^{2} \geq \frac{C_{5}^{\prime}}{K}\left[d\left(z, E_{\psi}\right)+d\left(w, E_{\psi}\right)\right]^{N_{2}} .
$$

Combining this with (3.8) completes the proof of Theorem 2. 


\section{REFERENCES}

[Eh] Leon Ehrenpreis, Solutions of some division problems I, American Journal of Mathematics 76 (1954), 883-903.

[EpHe] Charles L. Epstein and Gennadi M. Henkin, Stability of embeddings of 3-dimensional CR-manifolds, $I I$, in preparation (1998).

[GrHa] Phillip Griffiths and Joe Harris, Principles of Algebraic Geometry, Wiley-Interscience, New York, 1978.

[Hö] Lars Hörmander, On the division of distributions by polynomials, Ark. Mat. 3 (1958), 555-568.

[Ło] Stanislaw Łojasiewicz, Sur le problème de division, Studia Math. 18 (1959), 87-136.

[Ma] B. Malgrange, Existence et Approximation des solutions des équations aux dérivées partielles et des équations de convolution, Ann. Inst. Fourier Grenoble 6 (1955-6), 271-355. 